\title{
On approximation of hexagonal Fourier series in the generalized Hölder metric
}

\author{
Hatice Aslan ${ }^{1}$ (D), Ali Guven*2 (D) \\ ${ }^{1}$ Department of Mathematics, Faculty of Sciences, Firat University, 23000 Elazig, Turkey \\ ${ }^{2}$ Department of Mathematics, Faculty of Arts and Sciences, Balikesir University, 10145 Balikesir, Turkey
}

\begin{abstract}
Let $f$ be an $H$-periodic continuous function. The approximation order of the function $f$ by deferred Cesàro means of its hexagonal Fourier series is estimated in uniform and generalized Hölder metrics.
\end{abstract}

Mathematics Subject Classification (2010). 41A25, 42A10, 42B08

Keywords. deferred Cesàro means, generalized Hölder class, hexagonal Fourier series

\section{Introduction}

The order of approximation of $2 \pi$-periodic continuous functions on the real line is studied by many mathematicians in recent decades. There are several results on the order of approximation by partial sums and various means (Cesàro means, Abel-Poisson means, de la Vallèe-Poussin means, etc.) of trigonometric Fourier series in the literature. In these works, usually, it is assumed that the functions belong to Hölder spaces or their generalizations (see $[2,3,9-11,13,15,17]$ ).

Estimating the degree of approximation of functions of several real variables is also important in trigonometric approximation theory. Approximation problems of functions defined on cubes of the $d$-dimensional Euclidean space $\mathbb{R}^{d}$ are usually studied by assuming that the functions are $2 \pi$-periodic with respect to each of their variables (see, for example, [15, Sections 5.3 and 6.3] and [17, Vol. II, Chapter XVII]).

In the case of non-tensor product domains, for example, in the case of hexagonal domains in the Euclidean plane $\mathbb{R}^{2}$, other types of periodicity are needed to study approximation problems. The periodicity defined by lattices allows us to study approximation problems on spectral sets of lattices. In this section we give basic knowledge about hexagonal lattices, $H$-periodic functions and hexagonal Fourier series. It is well known that the hexagon lattice offers the densest packing of $\mathbb{R}^{2}$ with unit balls. Thus, the hexagon lattice and hexagonal Fourier series have great importance in Fourier analysis. More general information about lattices and Fourier analysis on spectral sets of them can be found in [12] and [16].

The hexagonal lattice is defined by

$$
H \mathbb{Z}^{2}:=\left\{H k: k \in \mathbb{Z}^{2}\right\},
$$

\footnotetext{
*Corresponding Author.

Email addresses: haticeaslan2017@gmail.com (H. Aslan), guvennali@gmail.com (A. Guven)

Received: 15.01.2019; Accepted: 28.05.2019
} 
where

$$
H=\left(\begin{array}{cc}
\sqrt{3} & 0 \\
-1 & 2
\end{array}\right)
$$

and $\mathbb{Z}^{2}=\left\{k=\left(k_{1}, k_{2}\right) \in \mathbb{R}^{2}: k_{1}, k_{2} \in \mathbb{Z}\right\} . H$ is called the generator matrix of this lattice. The spectral (or fundamental) set of hexagonal lattice is

$$
\Omega_{H}=\left\{\left(x_{1}, x_{2}\right) \in \mathbb{R}^{2}:-1 \leq x_{2}, \frac{\sqrt{3}}{2} x_{1} \pm \frac{1}{2} x_{2}<1\right\} .
$$

It is more convenient to use the homogeneous coordinates $\left(t_{1}, t_{2}, t_{3}\right)$ that satisfies $t_{1}+t_{2}+$ $t_{3}=0$. If we define

$$
t_{1}:=-\frac{x_{2}}{2}+\frac{\sqrt{3} x_{1}}{2}, t_{2}:=x_{2}, t_{3}:=-\frac{x_{2}}{2}-\frac{\sqrt{3} x_{1}}{2},
$$

the hexagon $\Omega_{H}$ becomes

$$
\Omega=\left\{\left(t_{1}, t_{2}, t_{3}\right) \in \mathbb{R}^{3}:-1 \leq t_{1}, t_{2},-t_{3}<1, t_{1}+t_{2}+t_{3}=0\right\},
$$

which is the intersection of the plane $t_{1}+t_{2}+t_{3}=0$ with the cube $[-1,1]^{3}$.

We use bold letters $\mathbf{t}$ for homogeneous coordinates and we denote by $\mathbb{R}_{H}^{3}$ the plane $t_{1}+t_{2}+t_{3}=0$, that is

$$
\mathbb{R}_{H}^{3}=\left\{\mathbf{t}=\left(t_{1}, t_{2}, t_{3}\right) \in \mathbb{R}^{3}: t_{1}+t_{2}+t_{3}=0\right\} .
$$

Also we use the notation $\mathbb{Z}_{H}^{3}$ for the set of points in $\mathbb{R}_{H}^{3}$ with integer components, that is $\mathbb{Z}_{H}^{3}=\mathbb{Z}^{3} \cap \mathbb{R}_{H}^{3}$.

$L^{2}(\Omega)$ is a Hilbert space with respect to the inner product

$$
\langle f, g\rangle_{H}=\frac{1}{|\Omega|} \int_{\Omega} f(\mathbf{t}) \overline{g(\mathbf{t})} d \mathbf{t}, \quad\left(f, g \in L^{2}(\Omega)\right),
$$

where $|\Omega|$ denotes the area of $\Omega$ and $\overline{g(\mathbf{t})}$ is the conjugate of $g(\mathbf{t})$. By Fuglede's theorem (see [4]) the set

$$
\left\{\phi_{\mathbf{j}}(\mathbf{t})=e^{\frac{2 \pi i}{3}\langle\mathbf{j}, \mathbf{t}\rangle}: \mathbf{j} \in \mathbb{Z}_{H}^{3}, \mathbf{t} \in \mathbb{R}_{H}^{3}\right\},
$$

where $\langle\mathbf{j}, \mathbf{t}\rangle$ is the usual Euclidean inner product of $\mathbf{j}$ and $\mathbf{t}$, is an orthonormal basis of this space.

A function $f$ is called periodic with respect to the hexagonal lattice (or $H$-periodic) if $f(\mathbf{t})=f(\mathbf{t}+\mathbf{s})$ whenever $\mathbf{s} \equiv \mathbf{0}(\bmod 3)$, where $\mathbf{t} \equiv \mathbf{s}(\bmod 3)$ defined as

$$
t_{1}-s_{1} \equiv t_{2}-s_{2} \equiv t_{3}-s_{3}(\bmod 3) \text {. }
$$

It is clear that the functions $\phi_{\mathbf{j}}(\mathbf{t})$ are $H$-periodic and if the function $f$ is $H$-periodic then

$$
\int_{\Omega} f(\mathbf{t}+\mathbf{s}) d \mathbf{t}=\int_{\Omega} f(\mathbf{t}) d \mathbf{t}, \quad\left(\mathbf{s} \in \mathbb{R}_{H}^{3}\right) .
$$

For every natural number $n$, we define a subset of $\mathbb{Z}_{H}^{3}$ by

$$
\mathbb{H}_{n}:=\left\{\mathbf{j}=\left(j_{1}, j_{2}, j_{3}\right) \in \mathbb{Z}_{H}^{3}:-n \leq j_{1}, j_{2}, j_{3} \leq n\right\} .
$$

Note that, $\mathbb{H}_{n}$ consists of all points with integer components inside the hexagon $n \bar{\Omega}$.

The hexagonal Fourier series of an $H$-periodic function $f \in L^{1}(\Omega)$ is

$$
f(\mathbf{t}) \sim \sum_{\mathbf{j} \in \mathbb{Z}_{H}^{3}} \widehat{f}_{\mathbf{j}} \phi_{\mathbf{j}}(\mathbf{t}),
$$

where

$$
\widehat{f_{\mathbf{j}}}=\frac{1}{|\Omega|} \int_{\Omega} f(\mathbf{t}) e^{-\frac{2 \pi i}{3}\langle\mathbf{j}, \mathbf{t}\rangle} d \mathbf{t}, \quad\left(\mathbf{j} \in \mathbb{Z}_{H}^{3}\right) .
$$


The $n$th partial sum of the series (1.2) is defined by

$$
S_{n}(f)(\mathbf{t}):=\sum_{\mathbf{j} \in \mathbb{H}_{n}} \widehat{f}_{\mathbf{j}} \phi_{\mathbf{j}}(\mathbf{t}),(n \in \mathbb{N}) .
$$

It is clear that

$$
S_{n}(f)(\mathbf{t})=\frac{1}{|\Omega|} \int_{\Omega} f(\mathbf{t}-\mathbf{s}) D_{n}(\mathbf{s}) d \mathbf{s},
$$

where

$$
D_{n}(\mathbf{t}):=\sum_{\mathbf{j} \in \mathbb{H}_{n}} \phi_{\mathbf{j}}(\mathbf{t})
$$

is the Dirichlet kernel.

The Dirichlet kernel has the compact formula

$$
D_{n}(\mathbf{t})=\Theta_{n}(\mathbf{t})-\Theta_{n-1}(\mathbf{t}), \quad(n \in \mathbb{N}),
$$

where

$$
\Theta_{n}(\mathbf{t}):=\frac{\sin \frac{(n+1)\left(t_{1}-t_{2}\right) \pi}{3} \sin \frac{(n+1)\left(t_{2}-t_{3}\right) \pi}{3} \sin \frac{(n+1)\left(t_{3}-t_{1}\right) \pi}{3}}{\sin \frac{\left(t_{1}-t_{2}\right) \pi}{3} \sin \frac{\left(t_{2}-t_{3}\right) \pi}{3} \sin \frac{\left(t_{3}-t_{1}\right) \pi}{3}}
$$

for $\mathbf{t}=\left(t_{1}, t_{2}, t_{3}\right) \in \mathbb{R}_{H}^{3}([14],[12])$.

\section{Main results}

Let $C_{H}(\bar{\Omega})$ be the space of complex valued $H$-periodic continuous functions defined on $\mathbb{R}_{H}^{3}$. $C_{H}(\bar{\Omega})$ is a Banach space with respect to the uniform norm

$$
\|f\|_{C(\bar{\Omega})}=\sup \{|f(\mathbf{t})|: \mathbf{t} \in \bar{\Omega}\} .
$$

A function $f \in C_{H}(\bar{\Omega})$ is said to belong to the Hölder space $H^{\alpha}(\bar{\Omega}), 0<\alpha \leq 1$, if

$$
\sup _{\mathbf{t} \neq \mathbf{s}} \frac{|f(\mathbf{t})-f(\mathbf{s})|}{\|\mathbf{t}-\mathbf{s}\|^{\alpha}}<\infty
$$

where $\|\mathbf{t}\|=\max \left\{\left|t_{1}\right|,\left|t_{2}\right|,\left|t_{3}\right|\right\}$.

In [16], the author proved that the Abel-Poisson means and the sequence of Cesàro $(C, 1)$ means of the Fourier series of a function $f \in C_{H}(\bar{\Omega})$ converge to this function uniformly on $\bar{\Omega}$. Later, the order of approximation by Abel-Poisson and $(C, 1)$ means of Fourier series of functions belong to the class $H^{\alpha}(\bar{\Omega})(0<\alpha \leq 1)$ were investigated in uniform norm ([6]) and in the Hölder norm ([5]).

Let $p=\left(p_{n}\right)$ and $q=\left(q_{n}\right)$ be two sequences of non-negative integers such that

$$
p_{n}<q_{n} \text { and } \lim _{n \rightarrow \infty} q_{n}=\infty .
$$

The deferred Cesàro means of the series (1.2) are defined by

$$
D_{n}(p, q ; f)(\mathbf{t}):=\frac{1}{q_{n}-p_{n}} \sum_{k=p_{n}+1}^{q_{n}} S_{k}(f)(\mathbf{t}) .
$$

It is known that the $D_{n}(p, q)$ summability method is regular under conditions in (2.1) and generalizes the Cesàro $(C, 1)$ method if and only if $p_{n} \lesssim q_{n}-p_{n}([1])$. 
By considering (1.3) and (1.4) we obtain

$$
\begin{aligned}
D_{n}(p, q ; f)(\mathbf{t}) & =\frac{1}{|\Omega|} \int_{\Omega} f(\mathbf{t}-\mathbf{s})\left(\frac{1}{q_{n}-p_{n}} \sum_{k=p_{n}+1}^{q_{n}} D_{k}(\mathbf{s})\right) d \mathbf{s} \\
& =\frac{1}{q_{n}-p_{n}} \frac{1}{|\Omega|} \int_{\Omega} f(\mathbf{t}-\mathbf{s})\left(\sum_{k=p_{n}+1}^{q_{n}} \Theta_{k}(\mathbf{s})-\Theta_{k-1}(\mathbf{s})\right) d \mathbf{s} \\
& =\frac{1}{q_{n}-p_{n}} \frac{1}{|\Omega|} \int_{\Omega} f(\mathbf{t}-\mathbf{s})\left(\Theta_{q_{n}}(\mathbf{s})-\Theta_{p_{n}}(\mathbf{s})\right) d \mathbf{s} .
\end{aligned}
$$

Hence we have

$$
f(\mathbf{t})-D_{n}(p, q ; f)(\mathbf{t})=\frac{1}{\left(q_{n}-p_{n}\right)} \frac{1}{|\Omega|} \int_{\Omega}(f(\mathbf{t})-f(\mathbf{t}-\mathbf{s}))\left(\Theta_{q_{n}}(\mathbf{s})-\Theta_{p_{n}}(\mathbf{s})\right) d \mathbf{s}
$$

for each $f \in L^{1}(\Omega)$ and $\mathbf{t} \in \mathbb{R}_{H}^{3}$.

If we take $q_{n}=n$ and $p_{n}=0$ for $n=1,2, \ldots, D_{n}(p, q ; f)$ become the $(C, 1)$ means $S_{n}^{(1)}(f)$.

Hereafter, we shall write $A \lesssim B$ for the quantities $A$ and $B$, if there exists a constant $K>0$ such that $A \leq K B$ holds.

The order of approximation by deferred Cesàro means of hexagonal Fourier series was estimated by the second author as follows [8].

Theorem 2.1. Let $f \in H^{\alpha}(\bar{\Omega}), 0<\alpha \leq 1$. Then the estimate

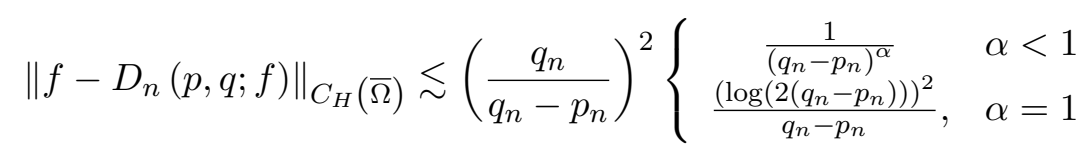

holds.

Theorem 2.2. Let $0 \leq \beta<\alpha \leq 1$ and $f \in H^{\alpha}(\bar{\Omega})$. Then we have

$$
\left\|f-D_{n}(p, q ; f)\right\|_{H^{\beta}(\bar{\Omega})} \lesssim\left(\frac{q_{n}}{q_{n}-p_{n}}\right)^{2}\left\{\begin{array}{cc}
\frac{1}{\left(q_{n}-p_{n}\right)^{\alpha-\beta}}, & \alpha<1 \\
\frac{\left(\log \left(2\left(q_{n}-p_{n}\right)\right)^{2}\right.}{\left(q_{n}-p_{n}\right)^{1-\beta}}, & \alpha=1 .
\end{array}\right.
$$

A non-decreasing continuous function $\omega:[0, \infty) \rightarrow[0, \infty)$ is called a modulus of continuity if

$$
\omega(0)=0, \omega\left(t_{1}+t_{2}\right) \leq \omega\left(t_{1}\right)+\omega\left(t_{2}\right) .
$$

For any modulus of continuity $\omega$, we define the generalized Hölder class $H^{\omega}(\bar{\Omega})$ as the set of functions $f \in C_{H}(\bar{\Omega})$ for which

$$
\sup _{\mathbf{t} \neq \mathbf{s}} \frac{|f(\mathbf{t})-f(\mathbf{s})|}{\omega(\|\mathbf{t}-\mathbf{s}\|)}<\infty
$$

and the norm on $H^{\omega}(\bar{\Omega})$ as

$$
\|f\|_{\omega}:=\|f\|_{C(\bar{\Omega})}+\sup _{\mathbf{t} \neq \mathbf{s}} \frac{|f(\mathbf{t})-f(\mathbf{s})|}{\omega(\|\mathbf{t}-\mathbf{s}\|)} .
$$

If $\omega(\delta)=\delta^{\alpha}, 0<\alpha \leq 1$, then it is clear that $H^{\omega}(\bar{\Omega})$ coincides with $H^{\alpha}(\bar{\Omega})$, and $\|f\|_{\omega}$ becomes $\|f\|_{\alpha}$.

In [10], L. Leindler introduced a certain class of moduli of continuity:

for $0 \leq \alpha \leq 1$, let $\mathcal{M}_{\alpha}$ denote the class of moduli of continuity $\omega_{\alpha}$ having the following properties: 
(i) for any $\alpha^{\prime}>\alpha$ there exists a natural number $\mu=\mu\left(\alpha^{\prime}\right)$ such that

$$
2^{\mu \alpha^{\prime}} \omega_{\alpha}\left(2^{-n-\mu}\right)>2 \omega_{\alpha}\left(2^{-n}\right), \quad(n=1,2, \ldots),
$$

(ii) for every natural number $\nu$, there exists a natural number $N(\nu)$ such that

$$
2^{\nu \alpha} \omega_{\alpha}\left(2^{-n-\nu}\right) \leq 2 \omega_{\alpha}\left(2^{-n}\right), \quad(n>N(\nu)) .
$$

It is clear that $\omega(\delta)=\delta^{\alpha} \in \mathcal{M}_{\alpha}$, but $\omega_{\alpha}(\delta)$ is an extension of $\omega(\delta)=\delta^{\alpha}$. Consequently, in general, $H^{\omega_{\alpha}}(\bar{\Omega})$ is larger than $H^{\alpha}(\bar{\Omega})$.

It is known that if $0 \leq \beta<\alpha \leq 1, \omega_{\beta} \in \mathcal{M}_{\beta}$ and $\omega_{\alpha} \in \mathcal{M}_{\alpha}$, then the function

$$
\gamma(t)=\gamma_{\alpha, \beta}(t):=\frac{\omega_{\alpha}(t)}{\omega_{\beta}(t)}
$$

is non-decreasing ([11]).

In this work, we will estimate the order of approximation by deferred Cesàro means of hexagonal Fourier series of functions belong to the generalized Hölder class $H^{\omega_{\alpha}}(\bar{\Omega})$ and generalize the some results of [6] and [5], [8].

Main results of this work are the following.

Theorem 2.3. Let $0 \leq \beta<\alpha \leq 1, \omega_{\beta} \in \mathcal{M}_{\beta}, \omega_{\alpha} \in \mathcal{M}_{\alpha}$ and $f \in H^{\omega_{\alpha}}(\bar{\Omega})$. Then

$$
\begin{aligned}
& \left\|f-D_{n}(p, q ; f)\right\|_{C_{H}(\bar{\Omega})}
\end{aligned}
$$

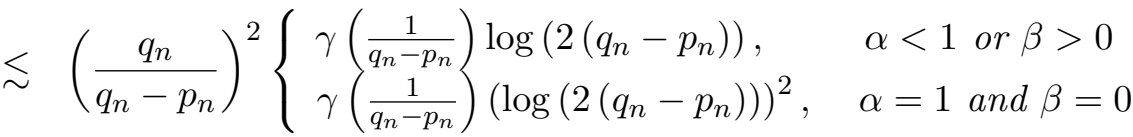

holds.

Theorem 2.4. Let $0 \leq \beta<\alpha \leq 1, \omega_{\beta} \in \mathcal{M}_{\beta}, \omega_{\alpha} \in \mathcal{M}_{\alpha}$ and $f \in H^{\omega_{\alpha}}(\bar{\Omega})$. Then

$$
\begin{aligned}
& \left\|f-D_{n}(p, q ; f)\right\|_{H^{\omega_{\beta}}(\bar{\Omega})} \\
\lesssim & \left(\frac{q_{n}}{q_{n}-p_{n}}\right)^{2}\left\{\begin{array}{l}
\gamma\left(\frac{1}{q_{n}-p_{n}}\right) \log \left(2\left(q_{n}-p_{n}\right)\right), \\
\gamma\left(\frac{1}{q_{n}-p_{n}}\right)\left(\log \left(2\left(q_{n}-p_{n}\right)\right)\right)^{2},
\end{array} \quad \alpha=1 \text { and } \beta=0 .\right.
\end{aligned}
$$

\section{Proofs of main results}

Proof of Theorem 2.3. Since $f \in H^{\omega_{\alpha}}(\bar{\Omega})$, by (2.2) we have,

$$
\left|f(\mathbf{t})-D_{n}(p, q ; f)(\mathbf{t})\right| \lesssim \frac{1}{\left(q_{n}-p_{n}\right)} \frac{1}{|\Omega|} \int_{\Omega} \omega_{\alpha}(\|\mathbf{s}\|)\left|\Theta_{q_{n}}(\mathbf{s})-\Theta_{p_{n}}(\mathbf{s})\right| d \mathbf{s} .
$$

Since the integrated function symmetric with respect to its variables, it is sufficient to estimate this integral

$$
I_{n}:=\int_{\Delta} \omega_{\alpha}(\|\mathbf{t}\|)\left|\Theta_{q_{n}}(\mathbf{t})-\Theta_{p_{n}}(\mathbf{t})\right| d \mathbf{t}
$$

where

$$
\begin{aligned}
\Delta & :=\left\{\mathbf{t}=\left(t_{1}, t_{2}, t_{3}\right) \in \mathbb{R}_{H}^{3}: 0 \leq t_{1}, t_{2},-t_{3} \leq 1\right\} \\
& =\left\{\left(t_{1}, t_{2}\right): t_{1} \geq 0, t_{2} \geq 0, t_{1}+t_{2} \leq 1\right\},
\end{aligned}
$$

which is one of the six equilateral triangles in $\bar{\Omega}$.

By (1.5) and some simple trigonometric identities,

$$
\begin{aligned}
& \Theta_{q_{n}}(\mathbf{t})-\Theta_{p_{n}}(\mathbf{t}) \\
= & \frac{2 \cos \left(\left(\frac{q_{n}+p_{n}}{2}+1\right) \frac{\left(t_{3}-t_{1}\right) \pi}{3}\right) \sin \left(\left(\frac{q_{n}-p_{n}}{2}\right) \frac{\left(t_{3}-t_{1}\right) \pi}{3}\right) \sin \left(\left(q_{n}+1\right) \frac{\left(t_{1}-t_{2}\right) \pi}{3}\right) \sin \left(\left(q_{n}+1\right) \frac{\left(t_{2}-t_{3}\right) \pi}{3}\right)}{\sin \frac{\left(t_{1}-t_{2}\right) \pi}{3} \sin \frac{\left(t_{2}-t_{3}\right) \pi}{3} \sin \frac{\left(t_{3}-t_{1}\right) \pi}{3}}
\end{aligned}
$$




$$
\begin{aligned}
& +\frac{2 \cos \left(\left(\frac{q_{n}+p_{n}}{2}+1\right) \frac{\left(t_{2}-t_{3}\right) \pi}{3}\right) \sin \left(\left(\frac{q_{n}-p_{n}}{2}\right) \frac{\left(t_{2}-t_{3}\right) \pi}{3}\right) \sin \left(\left(q_{n}+1\right) \frac{\left(t_{1}-t_{2}\right) \pi}{3}\right) \sin \left(\left(p_{n}+1\right) \frac{\left(t_{3}-t_{1}\right) \pi}{3}\right)}{\sin \frac{\left(t_{1}-t_{2}\right) \pi}{3} \sin \frac{\left(t_{2}-t_{3}\right) \pi}{3} \sin \frac{\left(t_{3}-t_{1}\right) \pi}{3}} \\
& +\frac{2 \cos \left(\left(\frac{q_{n}+p_{n}}{2}+1\right) \frac{\left(t_{1}-t_{2}\right) \pi}{3}\right) \sin \left(\left(\frac{q_{n}-p_{n}}{2}\right) \frac{\left(t_{1}-t_{2}\right) \pi}{3}\right) \sin \left(\left(p_{n}+1\right) \frac{\left(t_{2}-t_{3}\right) \pi}{3}\right) \sin \left(\left(p_{n}+1\right) \frac{\left(t_{3}-t_{1}\right) \pi}{3}\right)}{\sin \frac{\left(t_{1}-t_{2}\right) \pi}{3} \sin \frac{\left(t_{2}-t_{3}\right) \pi}{3} \sin \frac{\left(t_{3}-t_{1}\right) \pi}{3}} .
\end{aligned}
$$

If we use the change of variables

we obtain

$$
s_{1}:=\frac{t_{1}-t_{3}}{3}, s_{2}:=\frac{t_{2}-t_{3}}{3}
$$

$$
I_{n} \leq 3 \int_{\widetilde{\Delta}} \omega_{\alpha}\left(s_{1}+s_{2}\right)\left(\left|L_{1, n}\left(s_{1}, s_{2}\right)\right|+\left|L_{2, n}\left(s_{1}, s_{2}\right)\right|+\left|L_{3, n}\left(s_{1}, s_{2}\right)\right|\right) d s_{1} d s_{2}
$$

where

$$
\widetilde{\Delta}:=\left\{\left(s_{1}, s_{2}\right): 0 \leq s_{1} \leq 2 s_{2}, 0 \leq s_{2} \leq 2 s_{1}, s_{1}+s_{2} \leq 1\right\}
$$

and

$$
\begin{aligned}
& L_{1, n}\left(s_{1}, s_{2}\right):=\frac{\sin \left(\left(\frac{q_{n}-p_{n}}{2}\right)\left(s_{1} \pi\right)\right) \sin \left(\left(q_{n}+1\right)\left(s_{1}-s_{2}\right) \pi\right) \sin \left(\left(q_{n}+1\right) s_{2} \pi\right)}{\sin \left(\left(s_{1}-s_{2}\right) \pi\right) \sin \left(s_{2} \pi\right) \sin \left(s_{1} \pi\right)} \\
& L_{2, n}\left(s_{1}, s_{2}\right):=\frac{\sin \left(\left(\frac{q_{n}-p_{n}}{2}\right)\left(s_{2} \pi\right)\right) \sin \left(\left(q_{n}+1\right)\left(s_{1}-s_{2}\right) \pi\right) \sin \left(\left(p_{n}+1\right)\left(s_{1} \pi\right)\right)}{\sin \left(\left(s_{1}-s_{2}\right) \pi\right) \sin \left(s_{2} \pi\right) \sin \left(s_{1} \pi\right)} \\
& L_{3, n}\left(s_{1}, s_{2}\right):=\frac{\sin \left(\left(\frac{q_{n}-p_{n}}{2}\right)\left(s_{1}-s_{2}\right) \pi\right) \sin \left(\left(p_{n}+1\right)\left(s_{2} \pi\right)\right) \sin \left(\left(p_{n}+1\right)\left(s_{1} \pi\right)\right)}{\sin \left(\left(s_{1}-s_{2}\right) \pi\right) \sin \left(s_{2} \pi\right) \sin \left(s_{1} \pi\right)} .
\end{aligned}
$$

Since the integrated function symmetric with respect to $s_{1}$ and $s_{2}$, we have

$$
I_{n} \leq 6 \int_{\Delta^{*}} \omega_{\alpha}\left(s_{1}+s_{2}\right)\left(\left|L_{1, n}\left(s_{1}, s_{2}\right)\right|+\left|L_{2, n}\left(s_{1}, s_{2}\right)\right|+\left|L_{3, n}\left(s_{1}, s_{2}\right)\right|\right) d s_{1} d s_{2},
$$

where

$$
\Delta^{*}:=\left\{\left(s_{1}, s_{2}\right) \in \widetilde{\Delta}: s_{1} \leq s_{2}\right\},
$$

i. e. the half of $\widetilde{\Delta}$. The change of variables

$$
s_{1}:=\frac{u_{1}-u_{2}}{2}, s_{2}:=\frac{u_{1}+u_{2}}{2}
$$

transforms the triangle $\Delta^{*}$ onto triangle

and hence

$$
\Gamma:=\left\{\left(u_{1}, u_{2}\right): 0 \leq u_{2} \leq \frac{u_{1}}{3}, 0 \leq u_{1} \leq 1\right\}
$$

$$
I_{n} \leq 3 \int_{\Gamma} \omega_{\alpha}\left(u_{1}\right)\left(\left|L_{1, n}^{*}\left(u_{1}, u_{2}\right)\right|+\left|L_{2, n}^{*}\left(u_{1}, u_{2}\right)\right|+\left|L_{3, n}^{*}\left(u_{1}, u_{2}\right)\right|\right) d u_{1} d u_{2},
$$

where

$$
\begin{aligned}
& L_{1, n}^{*}\left(u_{1}, u_{2}\right):=\frac{\sin \left(\left(\frac{q_{n}-p_{n}}{2}\right) \frac{\left(u_{1}-u_{2}\right) \pi}{2}\right) \sin \left(\left(q_{n}+1\right) u_{2} \pi\right) \sin \left(\left(q_{n}+1\right) \frac{\left(u_{1}+u_{2}\right) \pi}{2}\right)}{\sin \left(u_{2} \pi\right) \sin \left(\frac{\left(u_{1}+u_{2}\right) \pi}{2}\right) \sin \left(\frac{\left(u_{1}-u_{2}\right) \pi}{2}\right)} \\
& L_{2, n}^{*}\left(u_{1}, u_{2}\right):=\frac{\sin \left(\left(\frac{q_{n}-p_{n}}{2}\right) \frac{\left(u_{1}+u_{2}\right) \pi}{2}\right) \sin \left(\left(q_{n}+1\right) u_{2} \pi\right) \sin \left(\left(p_{n}+1\right) \frac{\left(u_{1}-u_{2}\right) \pi}{2}\right)}{\sin \left(u_{2} \pi\right) \sin \left(\frac{\left(u_{1}+u_{2}\right) \pi}{2}\right) \sin \left(\frac{\left(u_{1}-u_{2}\right) \pi}{2}\right)} \\
& L_{3, n}^{*}\left(u_{1}, u_{2}\right):=\frac{\sin \left(\left(\frac{q_{n}-p_{n}}{2}\right) u_{2} \pi\right) \sin \left(\left(p_{n}+1\right) \frac{\left(u_{1}+u_{2}\right) \pi}{2}\right) \sin \left(\left(p_{n}+1\right) \frac{\left(u_{1}-u_{2}\right) \pi}{2}\right)}{\sin \left(u_{2} \pi\right) \sin \left(\frac{\left(u_{1}+u_{2}\right) \pi}{2}\right) \sin \left(\frac{\left(u_{1}-u_{2}\right) \pi}{2}\right)} .
\end{aligned}
$$


If we divide the triangle $\Gamma$ into three parts as

$$
\begin{aligned}
& \Gamma_{1}:=\left\{\left(u_{1}, u_{2}\right) \in \Gamma: u_{1} \leq \frac{1}{2\left(q_{n}-p_{n}\right)}\right\}, \\
& \Gamma_{2}: \quad=\left\{\left(u_{1}, u_{2}\right) \in \Gamma: u_{1} \geq \frac{1}{2\left(q_{n}-p_{n}\right)}, u_{2} \leq \frac{1}{6\left(q_{n}-p_{n}\right)}\right\}, \\
& \Gamma_{3}:=\left\{\left(u_{1}, u_{2}\right) \in \Gamma: u_{1} \geq \frac{1}{2\left(q_{n}-p_{n}\right)}, u_{2} \geq \frac{1}{6\left(q_{n}-p_{n}\right)}\right\},
\end{aligned}
$$

we get

$$
I_{n} \lesssim I_{1, n}+I_{2, n}+I_{3, n},
$$

where

$$
I_{j, n}=\int_{\Gamma_{j}} \omega_{\alpha}\left(u_{1}\right)\left(\left|L_{1, n}^{*}\left(u_{1}, u_{2}\right)\right|+\left|L_{2, n}^{*}\left(u_{1}, u_{2}\right)\right|+\left|L_{3, n}^{*}\left(u_{1}, u_{2}\right)\right|\right) d u_{1} d u_{2} \quad(j=1,2,3) .
$$

We use the inequalities

$$
\left|\frac{\sin n t}{\sin t}\right| \leq n, \quad(n \in \mathbb{N})
$$

and

$$
\sin t \geq \frac{2}{\pi} t, \quad\left(0 \leq t \leq \frac{\pi}{2}\right)
$$

to estimate integrals $I_{j, n}(j=1,2,3)$.

For $\left(u_{1}, u_{2}\right) \in \Gamma$, since $u_{2} \leq 1 / 3$, we have

$$
\begin{aligned}
\sin \left(\frac{u_{1}+u_{2}}{2} \pi\right) & =\sin \left(\frac{u_{1} \pi}{2}\right) \cos \left(\frac{u_{2} \pi}{2}\right)+\cos \left(\frac{u_{1} \pi}{2}\right) \sin \left(\frac{u_{2} \pi}{2}\right) \\
& \geq \sin \left(\frac{u_{1} \pi}{2}\right) \cos \left(\frac{u_{2} \pi}{2}\right) \geq \cos \left(\frac{\pi}{6}\right) \sin \left(\frac{u_{1} \pi}{2}\right)=\frac{\sqrt{3}}{2} \sin \frac{u_{1} \pi}{2}
\end{aligned}
$$

and by (3.4) we obtain

$$
\frac{1}{\sin \left(\frac{u_{1}+u_{2}}{2} \pi\right)} \leq \frac{2}{\sqrt{3}} \frac{1}{u_{1}}\left(u_{1} \neq 0\right) .
$$

By the inequality (3.3) we get

$$
\begin{aligned}
&\left|L_{1, n}^{*}\left(u_{1}, u_{2}\right)\right| \lesssim\left(q_{n}-p_{n}\right)\left(q_{n}+1\right)^{2} \\
&\left|L_{2, n}^{*}\left(u_{1}, u_{2}\right)\right| \lesssim\left(q_{n}-p_{n}\right)\left(q_{n}+1\right)\left(p_{n}+1\right) \\
&\left|L_{3, n}^{*}\left(u_{1}, u_{2}\right)\right| \lesssim\left(q_{n}-p_{n}\right)\left(p_{n}+1\right)^{2}
\end{aligned}
$$

and hence

$$
\left|L_{j, n}^{*}\left(u_{1}, u_{2}\right)\right| \lesssim\left(q_{n}-p_{n}\right) q_{n}^{2}(j=1,2,3)
$$


for $\left(u_{1}, u_{2}\right) \in \Gamma_{1}$. Thus,

$$
\begin{aligned}
I_{1, n} & \lesssim\left(q_{n}-p_{n}\right) q_{n}^{2} \int_{\Gamma_{1}} \omega_{\alpha}\left(u_{1}\right) d u_{1} d u_{2} \\
& =\left(q_{n}-p_{n}\right) q_{n}^{2} \int_{0}^{\frac{1}{2\left(q_{n}-p_{n}\right)} u_{1} / 3} \int_{0} \omega_{\alpha}\left(u_{1}\right) d u_{2} d u_{1} \\
& =\frac{\left(q_{n}-p_{n}\right) q_{n}^{2}}{3} \int_{0}^{\frac{1}{2\left(q_{n}-p_{n}\right)}} u_{1} \omega_{\alpha}\left(u_{1}\right) d u_{1} \\
& \leq\left(q_{n}-p_{n}\right) q_{n}^{2} \omega_{\alpha}\left(\frac{1}{2\left(q_{n}-p_{n}\right)}\right) \int_{0}^{\frac{1}{2\left(q_{n}-p_{n}\right)}} u_{1} d u_{1} \\
& \leq \frac{q_{n}^{2}}{q_{n}-p_{n}} \omega_{\alpha}\left(\frac{1}{q_{n}-p_{n}}\right) \\
& =\frac{q_{n}^{2}}{q_{n}-p_{n}} \omega_{\beta}\left(\frac{1}{q_{n}-p_{n}}\right) \gamma\left(\frac{1}{q_{n}-p_{n}}\right) \\
& \leq \omega_{\beta}(1) \frac{q_{n}^{2}}{q_{n}-p_{n}} \gamma\left(\frac{1}{q_{n}-p_{n}}\right) .
\end{aligned}
$$

Since $\left(u_{1}-u_{2}\right) \frac{\pi}{2} \leq \frac{\pi}{2}$, by (3.4) we get

$$
u_{1}-u_{2} \leq \sin \left(u_{1}-u_{2}\right) \frac{\pi}{2} .
$$

This inequality and the fact $u_{1}-u_{2} \geq \frac{2 u_{1}}{3}$ yield

$$
\frac{1}{\sin \left(\frac{\left(u_{1}-u_{2}\right) \pi}{2}\right)} \leq \frac{3}{2 u_{1}}, \quad\left(u_{1}, u_{2}\right) \in \Gamma_{2} \cup \Gamma_{3} .
$$

Thus, by (3.3), (3.5) and (3.6) we obtain

$$
\begin{aligned}
&\left|L_{1, n}^{*}\left(u_{1}, u_{2}\right)\right| \lesssim\left(q_{n}+1\right) \frac{1}{u_{1}^{2}}, \\
&\left|L_{2, n}^{*}\left(u_{1}, u_{2}\right)\right| \lesssim\left(q_{n}+1\right) \frac{1}{u_{1}^{2}}
\end{aligned}
$$

and

$$
\left|L_{3, n}^{*}\left(u_{1}, u_{2}\right)\right| \lesssim\left(q_{n}-p_{n}\right) \frac{1}{u_{1}^{2}}
$$

which implies

$$
\left|L_{j, n}^{*}\left(u_{1}, u_{2}\right)\right| \lesssim \frac{q_{n}}{u_{1}^{2}}(j=1,2,3)
$$

for $\left(u_{1}, u_{2}\right) \in \Gamma_{2}$.

By Lemma 1 of [7] we have

$$
\int_{1 / n}^{1} \frac{\omega_{\alpha}(t)}{t^{2} \omega_{\beta}(t)} d t \lesssim\left\{\begin{array}{cc}
n \gamma\left(\frac{1}{n}\right), & \alpha<1 \text { or } \beta>0 \\
n \gamma\left(\frac{1}{n}\right) \log n, & \alpha=1 \text { and } \beta=0
\end{array}\right.
$$


for every natural number $n \geq 2$. Hence we get

$$
\begin{aligned}
I_{2, n} & \lesssim q_{n} \int_{\frac{1}{2\left(q_{n}-p_{n}\right)}}^{1} \int_{0}^{\frac{1}{6\left(q_{n}-p_{n}\right)}} \frac{\omega_{\alpha}\left(u_{1}\right)}{u_{1}^{2}} d u_{2} d u_{1} \\
& =\frac{q_{n}}{6\left(q_{n}-p_{n}\right)} \int_{\frac{1}{2\left(q_{n}-p_{n}\right)}}^{1} \frac{\omega_{\alpha}\left(u_{1}\right)}{u_{1}^{2} \omega_{\beta}\left(u_{1}\right)} \omega_{\beta}\left(u_{1}\right) d u_{1} \\
& \leq \omega_{\beta}(1) \frac{q_{n}}{q_{n}-p_{n}} \int_{\frac{1}{2\left(q_{n}-p_{n}\right)}} \frac{\omega_{\alpha}\left(u_{1}\right)}{u_{1}^{2} \omega_{\beta}\left(u_{1}\right)} d u_{1} \\
& \lesssim q_{n}\left\{\begin{array}{c}
\gamma\left(\frac{1}{q_{n}-p_{n}}\right), \\
\gamma\left(\frac{1}{q_{n}-p_{n}}\right) \log \left(2\left(q_{n}-p_{n}\right)\right), \quad \alpha=1 \text { and } \beta=0 .
\end{array}\right.
\end{aligned}
$$

By (3.4), (3.5) and (3.6) we obtain

$$
\left|L_{j, n}^{*}\left(u_{1}, u_{2}\right)\right| \lesssim \frac{1}{u_{1}^{2} u_{2}}(j=1,2,3)
$$

for $\left(u_{1}, u_{2}\right) \in \Gamma_{3}$. Thus, by (3.7), we have

$$
\begin{aligned}
& I_{3, n} \lesssim \int_{\frac{1}{2\left(q_{n}-p_{n}\right)}}^{1} \int_{\frac{1}{6\left(q_{n}-p_{n}\right)}}^{u_{1} / 3} \frac{\omega_{\alpha}\left(u_{1}\right)}{u_{1}^{2} u_{2}} d u_{2} d u_{1} \\
& =\int_{\frac{1}{2\left(q_{n}-p_{n}\right)}}^{1} \frac{\omega_{\alpha}\left(u_{1}\right)}{u_{1}^{2}} \log \left(2\left(q_{n}-p_{n}\right) u_{1}\right) d u_{1} \\
& \leq \log \left(2\left(q_{n}-p_{n}\right)\right) \int_{\frac{1}{2\left(q_{n}-p_{n}\right)}}^{1} \frac{\omega_{\alpha}\left(u_{1}\right)}{u_{1}^{2}} d u_{1} \\
& =\log \left(2\left(q_{n}-p_{n}\right)\right) \int_{\frac{1}{2\left(q_{n}-p_{n}\right)}}^{1} \frac{\omega_{\alpha}\left(u_{1}\right)}{u_{1}^{2} \omega_{\beta}\left(u_{1}\right)} \omega_{\beta}\left(u_{1}\right) d u_{1} \\
& \leq \omega_{\beta}(1) \log \left(2\left(q_{n}-p_{n}\right)\right) \int_{\frac{1}{2\left(q_{n}-p_{n}\right)}}^{1} \frac{\omega_{\alpha}\left(u_{1}\right)}{u_{1}^{2} \omega_{\beta}\left(u_{1}\right)} d u_{1} \\
& \lesssim\left(q_{n}-p_{n}\right)\left\{\begin{array}{cc}
\gamma\left(\frac{1}{q_{n}-p_{n}}\right) \log \left(2\left(q_{n}-p_{n}\right)\right), & \alpha<1 \text { or } \beta>0 \\
\gamma\left(\frac{1}{q_{n}-p_{n}}\right)\left(\log \left(2\left(q_{n}-p_{n}\right)\right)\right)^{2}, & \alpha=1 \text { and } \beta=0 .
\end{array}\right.
\end{aligned}
$$

Combining these estimates of $I_{1, n}, I_{2, n}$ and $I_{3, n}$ finishes proof of Theorem 2.3

Proof of Theorem 2.4. Set $e_{n}(\mathbf{t}):=f(\mathbf{t})-D_{n}(p, q ; f)(\mathbf{t})$. Hence

$$
\left\|f-D_{n}(p, q ; f)\right\|_{H^{\omega_{\beta}}(\bar{\Omega})}=\left\|e_{n}\right\|_{C_{H}(\bar{\Omega})}+\sup _{\mathbf{t} \neq \mathbf{s}} \frac{\left|e_{n}(\mathbf{t})-e_{n}(\mathbf{s})\right|}{\omega_{\beta}(\|\mathbf{t}-\mathbf{s}\|)} .
$$


By (2.2) we have

$$
e_{n}(\mathbf{t})-e_{n}(\mathbf{s})=\frac{1}{\left(q_{n}-p_{n}\right)} \frac{1}{|\Omega|} \int_{\Omega} \varphi_{\mathbf{t}, \mathbf{s}}(\mathbf{u})\left(\Theta_{q_{n}}(\mathbf{u})-\Theta_{p_{n}}(\mathbf{u})\right) d \mathbf{u},
$$

where

$$
\varphi_{\mathbf{t}, \mathbf{s}}(\mathbf{u}):=f(\mathbf{t})-f(\mathbf{t}-\mathbf{u})-f(\mathbf{s})+f(\mathbf{s}-\mathbf{u}) .
$$

Thus,

$$
\left|e_{n}(\mathbf{t})-e_{n}(\mathbf{s})\right| \leq \frac{1}{q_{n}-p_{n}} \frac{1}{|\Omega|} \int_{\Omega}\left|\varphi_{\mathbf{t}, \mathbf{s}}(\mathbf{u})\right|\left|\left(\Theta_{q_{n}}(\mathbf{u})-\Theta_{p_{n}}(\mathbf{u})\right)\right| d \mathbf{u} .
$$

Since $f \in H^{\omega_{\alpha}}(\bar{\Omega})$, by using the inequality

$$
\left|\varphi_{\mathbf{t}, \mathbf{s}}(\mathbf{u})\right| \lesssim \omega_{\beta}(\|\mathbf{t}-\mathbf{s}\|) \frac{\omega_{\alpha}(\|\mathbf{u}\|)}{\omega_{\beta}(\|\mathbf{u}\|)}
$$

which is proved in [7],

$$
\left|e_{n}(\mathbf{t})-e_{n}(\mathbf{s})\right| \lesssim \frac{1}{q_{n}-p_{n}} \omega_{\beta}(\|\mathbf{t}-\mathbf{s}\|) \frac{1}{|\Omega|} \int_{\Omega} \frac{\omega_{\alpha}(\|\mathbf{u}\|)}{\omega_{\beta}(\|\mathbf{u}\|)}\left|\left(\Theta_{q_{n}}(\mathbf{u})-\Theta_{p_{n}}(\mathbf{u})\right)\right| d \mathbf{u} .
$$

As in proof of Theorem 2.3, it is sufficient to estimate the integral

$$
\int_{\Delta} \frac{\omega_{\alpha}(\|\mathbf{t}\|)}{\omega_{\beta}(\|\mathbf{t}\|)}\left|\left(\Theta_{q_{n}}(\mathbf{t})-\Theta_{p_{n}}(\mathbf{t})\right)\right| d \mathbf{t} .
$$

By the transforms (3.1) and (3.2),

$$
\begin{aligned}
& \int_{\Delta}\left|\left(\Theta_{q_{n}}(\mathbf{t})-\Theta_{p_{n}}(\mathbf{t})\right)\right| d \mathbf{t} \\
\lesssim & \int_{\Gamma} \frac{\omega_{\alpha}\left(u_{1}\right)}{\omega_{\beta}\left(u_{1}\right)}\left(\left|L_{1, n}^{*}\left(u_{1}, u_{2}\right)\right|+\left|L_{2, n}^{*}\left(u_{1}, u_{2}\right)\right|+\left|L_{3, n}^{*}\left(u_{1}, u_{2}\right)\right|\right) d u_{1} d u_{2} .
\end{aligned}
$$

Since

we get

$$
\left|L_{j, n}^{*}\left(u_{1}, u_{2}\right)\right| \lesssim\left(q_{n}-p_{n}\right) q_{n}^{2}, \quad\left(u_{1}, u_{2}\right) \in \Gamma_{1},
$$

$$
\begin{aligned}
& \int_{\Gamma_{1}} \frac{\omega_{\alpha}\left(u_{1}\right)}{\omega_{\beta}\left(u_{1}\right)}\left|L_{j, n}^{*}\left(u_{1}, u_{2}\right)\right| d u_{1} d u_{2} \lesssim\left(q_{n}-p_{n}\right) q_{n}^{2} \int_{0}^{\frac{1}{2\left(q_{n}-p_{n}\right)}}\left(\int_{0}^{\frac{u_{1}}{3}} \frac{\omega_{\alpha}\left(u_{1}\right)}{\omega_{\beta}\left(u_{1}\right)} d u_{2}\right) d u_{1} \\
& \leq\left(q_{n}-p_{n}\right) q_{n}^{2} \int_{0}^{\frac{1}{2\left(q_{n}-p_{n}\right)}} u_{1} \frac{\omega_{\alpha}\left(u_{1}\right)}{\omega_{\beta}\left(u_{1}\right)} d u_{2} d u_{1} \\
& \leq\left(q_{n}-p_{n}\right) q_{n}^{2} \frac{\omega_{\alpha}\left(1 / 2\left(q_{n}-p_{n}\right)\right)}{\omega_{\beta}\left(1 / 2\left(q_{n}-p_{n}\right)\right)} \int_{0}^{\frac{1}{2\left(q_{n}-p_{n}\right)}} u_{1} d u_{1} \\
& \lesssim \frac{q_{n}^{2}}{q_{n}-p_{n}} \gamma\left(\frac{1}{q_{n}-p_{n}}\right)
\end{aligned}
$$

for $j=1,2,3$.

Since

$$
\left|L_{j, n}^{*}\left(u_{1}, u_{2}\right)\right| \lesssim \frac{q_{n}}{u_{1}^{2}}(j=1,2,3)
$$


for $\left(u_{1}, u_{2}\right) \in \Gamma_{2}$, the inequality 3.7 gives

$$
\begin{aligned}
& \int_{\Gamma_{2}} \frac{\omega_{\alpha}\left(u_{1}\right)}{\omega_{\beta}\left(u_{1}\right)}\left|L_{j, n}^{*}\left(u_{1}, u_{2}\right)\right| d u_{1} d u_{2} \\
\lesssim & q_{n} \int_{0}^{\frac{1}{6\left(q_{n}-p_{n}\right)}} \int_{\frac{1}{2\left(q_{n}-p_{n}\right)}}^{1} \frac{\omega_{\alpha}\left(u_{1}\right)}{u_{1}^{2} \omega_{\beta}\left(u_{1}\right)} d u_{1} d u_{2} \\
\lesssim & q_{n}\left\{\begin{array}{cc}
\gamma\left(\frac{1}{q_{n}-p_{n}}\right), & \alpha<1 \text { or } \beta>0 \\
\log \left(2\left(q_{n}-p_{n}\right)\right) \gamma\left(\frac{1}{q_{n}-p_{n}}\right), & \alpha=1 \text { and } \beta=0 .
\end{array}\right.
\end{aligned}
$$

Also,

$$
\left|L_{j, n}^{*}\left(u_{1}, u_{2}\right)\right| \lesssim \frac{1}{u_{1}^{2} u_{2}}, \quad\left(u_{1}, u_{2}\right) \in \Gamma_{3}(j=1,2,3)
$$

and (3.7) yield

$$
\begin{aligned}
& \int_{\Gamma_{3}} \frac{\omega_{\alpha}\left(u_{1}\right)}{\omega_{\beta}\left(u_{1}\right)}\left|L_{j, n}^{*}\left(u_{1}, u_{2}\right)\right| d u_{1} d u_{2} \\
\lesssim & \int_{\frac{1}{2\left(q_{n}-p_{n}\right)}}^{1} \int_{\frac{1}{6\left(q_{n}-p_{n}\right)}}^{u_{1} / 3} \frac{1}{u_{1}^{2} u_{2}} \frac{\omega_{\alpha}\left(u_{1}\right)}{\omega_{\beta}\left(u_{1}\right)} d u_{2} d u_{1} \\
= & \int_{1}^{1} \frac{\omega_{\alpha}\left(u_{1}\right)}{u_{1}^{2} \omega_{\beta}\left(u_{1}\right)} \log \left(2 u_{1}\left(q_{n}-p_{n}\right)\right) d u_{1} \\
\lesssim & \log \left(2\left(q_{n}-p_{n}\right)\right) \int_{1}^{\frac{1}{2\left(q_{n}-p_{n}\right)}} \frac{\omega_{\alpha}\left(u_{1}\right)}{u_{1}^{2} \omega_{\beta}\left(u_{1}\right)} d u_{1} \\
\lesssim & q_{n} \log \left(2\left(q_{n}-p_{n}\right)\right)\left\{\begin{array}{c}
2\left(q_{n}-p_{n}\right) \\
\gamma\left(\frac{1}{q_{n}-p_{n}}\right) \log \left(2\left(q_{n}-p_{n}\right)\right), \quad \alpha=1 \text { and } \beta=0
\end{array}\right.
\end{aligned}
$$

for $j=1,2,3$.

By combining these inequalities we obtain

$\int_{\Delta} \frac{\omega_{\alpha}(\|\mathbf{t}\|)}{\omega_{\beta}(\|\mathbf{t}\|)}\left|\left(\Theta_{q_{n}}(\mathbf{t})-\Theta_{p_{n}}(\mathbf{t})\right)\right| d \mathbf{t} \lesssim \frac{q_{n}^{2}}{q_{n}-p_{n}}\left\{\begin{array}{cc}\gamma\left(\frac{1}{q_{n}-p_{n}}\right) \log \left(2\left(q_{n}-p_{n}\right)\right), & \alpha<1 \text { or } \beta>0 \\ \gamma\left(\frac{1}{q_{n}-p_{n}}\right) \log \left(2\left(q_{n}-p_{n}\right)\right)^{2}, & \alpha=1 \text { and } \beta=0\end{array}\right.$.

This implies

$\left|e_{n}(\mathbf{t})-e_{n}(\mathbf{s})\right| \lesssim\left(\frac{q_{n}}{q_{n}-p_{n}}\right)^{2} \omega_{\beta}(\|\mathbf{t}-\mathbf{s}\|)\left\{\begin{array}{cc}\gamma\left(\frac{1}{q_{n}-p_{n}}\right) \log \left(2\left(q_{n}-p_{n}\right)\right), & \alpha<1 \text { or } \beta>0 \\ \gamma\left(\frac{1}{q_{n}-p_{n}}\right) \log \left(2\left(q_{n}-p_{n}\right)\right)^{2}, & \alpha=1 \text { and } \beta=0\end{array}\right.$,

and hence

$$
\begin{aligned}
& \frac{\left|e_{n}(\mathbf{t})-e_{n}(\mathbf{s})\right|}{\omega_{\beta}(\|\mathbf{t}-\mathbf{s}\|)}
\end{aligned}
$$

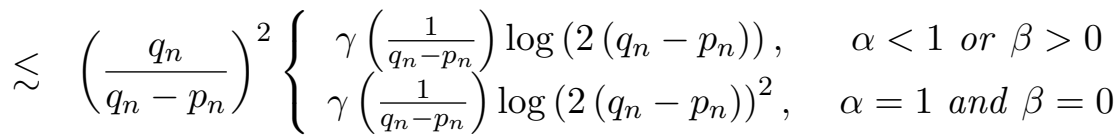

for every $\mathbf{t}, \mathbf{s} \in \mathbb{R}_{H}^{3}$ with $\mathbf{t} \neq \mathbf{s}$. This estimate and Theorem 2.3 give the desired result. 


\section{References}

[1] R.P. Agnew, On deferred Cesàro means, Ann. of Math. 33 (3), 413-421, 1932.

[2] J. Bustamante and M.A. Jimenez, Trends in Hölder approximation, in: Approximation, Optimization and Mathematical Economics, 81-95, Springer, 2001.

[3] R.A. DeVore and G.G. Lorentz, Constructive Approximation, Springer-Verlag, 1993.

[4] B. Fuglede, Commuting self-adjoint partial differential operators and a group theoretic problem, J. Funct. Anal. 16, 101-121, 1974.

[5] A. Guven, Approximation by means of hexagonal Fourier series in Hölder norms, J. Class. Anal. 1, 43-52, 2012.

[6] A. Guven, Approximation by $(C, 1)$ and Abel-Poisson means of Fourier series on hexagonal domains, Math. Inequal. Appl. 16, 175-191, 2013.

[7] A. Guven, Approximation properties of hexagonal Fourier series in the generalized Hölder metric, Comput. Methods Funct. Theory, 13, 509-531, 2013.

[8] A. Guven, On approximation of hexagonal Fourier series, Azerb. J. Math. 8, 52-68, 2018.

[9] A.S.B. Holland, A survey of degree of approximation of continuous functions, SIAM Rev. 23, 344-379, 1981.

[10] L. Leindler, Generalizations of Prössdorf's theorems, Studia Sci. Math. Hungar. 14, 431-439, 1979.

[11] L. Leindler, A relaxed estimate of the degree of approximation by Fourier series in generalized Hölder metric, Anal. Math. 35, 51-60, 2009.

[12] H. Li, J. Sun and Y. Xu, Discrete Fourier analysis, cubature and interpolation on a hexagon and a triangle, SIAM J. Numer. Anal. 46, 1653-1681, 2008.

[13] S. Prössdorf, Zur konvergenz der Fourierreihen hölderstetiger funktionen, Math. Nachr. 69, 7-14, 1975.

[14] J. Sun, Multivariate Fourier series over a class of non tensor-product partition domains, J. Comput. Math. 21, 53-62, 2003.

[15] A.F. Timan, Theory of Approximation of Functions of a Real Variable, Pergamon Press, 1963.

[16] Y. Xu, Fourier series and approximation on hexagonal and triangular domains, Constr. Approx. 31, 115-138, 2010.

[17] A. Zygmund, Trigonometric Series, Cambridge Univ. Press, 1959. 